\title{
ISOCLINIC SUBSPACES AND QUANTUM ERROR CORRECTION
}

\author{
David W. Kribs, DaVid Mammarella And Rajesh Pereira
}

Abstract. We exhibit equivalent conditions for subspaces of an inner product space to be isoclinic, including a characterization based on the classical notion of canonical angles. We identify a connection with quantum error correction, showing that every quantum error correcting code is associated with a family of isoclinic subspaces, and we prove a converse for pairs of such subspaces. We also show how the canonical angles for isoclinic subspaces arise in the structure of the higher rank numerical ranges of the corresponding orthogonal projections.

Mathematics subject classification (2020): 15B99, 46C05, 47A12, 81P45, 94A40.

Keywords and phrases: Canonical angles, isoclinic subspaces, quantum error correcting codes, higher rank numerical ranges.

\section{REFERENCES}

[1] IgOR BALla AND BENNy SudAKov, Equiangular subspaces in Euclidean spaces, Discrete \& Computational Geometry 61 (2019), no. 1, 81-90.

[2] Charles H. Bennett, David P. DiVincenzo, John A. Smolin and William K. Wootters, Mixed state entanglement and quantum error correction, Physical Review A 54 (1996), 3824.

[3] AKe BJÖRCK AND GENE H. Golub, Numerical methods for computing angles between linear subspaces, Mathematics of Computation 27 (1973), no. 123, 579-594.

[4] Man-Duen Choi, Michael Giesinger, John A. Holbrook and David W. Kribs, Geometry of higher-rank numerical ranges, Linear and Multilinear Algebra 56 (2008), no. 1-2, 53-64.

[5] Man-Duen Choi, David W. KRIBS And Karol ŻYCZKows Ki, Higher-rank numerical ranges and compression problems, Linear Algebra and its Applications 418 (2006), no. 2-3, 828-839.

[6] Man-Duen Choi, David W. KRIBS And Karol ŻYCZKows Ki, Quantum error correcting codes from the compression formalism, Reports on Mathematical Physics 58 (2006), no. 1, 77-91.

[7] Hwa-Long Gau, Chi-Kwong Li, Yiu-Tung Poon and Nung-Sing Sze, Higher rank numerical ranges of normal matrices, SIAM Journal of Matrix Analysis and its Applications 32 (2011), $23-43$.

[8] Daniel Gottesman, Class of quantum error-correcting codes saturating the quantum Hamming bound, Physical Review A 54 (1996), 1862.

[9] S. G. Hoggar, New sets of equi-isoclinic n-planes from old, Proceedings of the Edinburgh Mathematical Society 20 (1977), no. 4, 287-291.

[10] Camille Jordan, Essai sur la géométrie à $n$ dimensions, Bulletin de la Société Mathématique de France 3 (1875), 103-174.

[11] Emanuel Knill and Raymond Laflamme, Theory of quantum error-correcting codes, Physical Review A 55 (1997), 900.

[12] Emanuel Knill, Raymond Laflamme and Lorenza Viola, Theory of quantum error correction for general noise, Physical Review Letters 84 (2000), no. 11, 2525.

[13] DAVID W. KRIBS, A quantum computing primer for operator theorists, Linear Algebra and its Applications 400 (2005), 147-167.

[14] Chi-Kwong Li And Yiu-Tung Poon, Generalized numerical ranges and quantum error correction, Journal of Operator Theory (2011), 335-351.

[15] Chi-Kwong Li, Yiu-Tung Poon And Nung-Sing Sze, Higher rank numerical ranges and low rank perturbations of quantum channels, Journal of Mathematical Analysis and Applications 348 (2008), no. 2, 843-855. 
[16] Chi-Kwong Li, Yiu-Tung Poon And Nung-Sing Sze, Condition for the higher rank numerical range to be non-empty, Linear and Multilinear Algebra 57 (2009), no. 4, 365-368.

[17] Chi-Kwong Li AND Nung-Sing Sze, Canonical forms, higher rank numerical ranges, totally isotropic subspaces, and matrix equations, Proceedings of the American Mathematical Society 136 (2008), no. 9, 3013-3023.

[18] Ruben A. Martinez-Avendano, Higher-rank numerical range in infinite-dimensional Hilbert space, Operators and Matrices 2 (2008), no. 2, 249-264.

[19] Michael A. Nielsen And IsaAC Chuang, Quantum Computation and Quantum Information, Cambridge University Press, 2000.

[20] PETER W. SHOR, Scheme for reducing decoherence in quantum computing memory, Physical Review A 52 (1995), R2493.

[21] ANDREw M. STEANE, Error correcting codes in quantum theory, Physical Review Letters 77 (1996), no. 5,793 .

[22] Hugo J. Woerdeman, The higher rank numerical range is convex, Linear and Multilinear Algebra 56 (2008), no. 1-2, 65-67.

[23] Yung-Chow Wong, Clifford parallels in elliptic $(2 n-1)$-spaces and isoclinic n-planes in Euclidean $2 n$-space, Bulletin of the American Mathematical Society 66 (1960), no. 4, $289-293$.

[24] Yung-Chow Wong, Linear geometry in Euclidean 4-space, no. 1, Southeast Asian Mathematical Society, 1977. 